\title{
Jets, Bulk Matter, and their Interaction in Heavy Ion Collisions at Several TeV
}

\author{
K. Werner ${ }^{(a)}$, Iu. Karpenko ${ }^{(b)}$, M. Bleicher ${ }^{(c)}$, T. Pierog ${ }^{(d)}$, S. Porteboeuf-Houssais ${ }^{(e)}$ \\ (a) SUBATECH, University of Nantes - IN2P3/CNRS-EMN, Nantes, France \\ (b) Bogolyubov Institute for Theoretical Physics, Kiev 143, 03680, Ukraine \\ (c) FIAS, Johann Wolfgang Goethe Universitaet, Frankfurt am Main, Germany \\ ${ }^{(d)}$ Karlsruhe Inst. of Technology, KIT, Campus North, Inst. f. Kernphysik, Germany and \\ (e) LPC, Université Blaise Pascal, CNRS-IN2P3, Clermont-Ferrand, France
}

\begin{abstract}
We discuss a theoretical scheme that accounts for bulk matter, jets, and the interaction between the two. The aim is a complete description of particle production at all transverse momentum $\left(p_{t}\right)$ scales. In this picture, the hard initial scatterings result in mainly longitudinal flux tubes, with transversely moving pieces carrying the $p_{t}$ of the partons from hard scatterings. These flux tubes constitute eventually both bulk matter (which thermalizes and flows) and jets. We introduce a criterion based on parton energy loss to decide whether a given string segment contributes to the bulk or leaves the matter to end up as a jet of hadrons. Essentially low $p_{t}$ segments from inside the volume will constitute the bulk, high $p_{t}$ segments (or segments very close to the surface) contribute to the jets. The latter ones appear after the usual flux tube breaking via q-qbar production (Schwinger mechanism). Interesting is the transition region: Intermediate $p_{t}$ segments produced inside the matter close to the surface but having enough energy to escape, are supposed to pick up q-qbar pairs from the thermal matter rather than creating them via the Schwinger mechanism. This represents a communication between jets and the flowing bulk matter (fluid-jet interaction). Also very important is the interaction between jet hadrons and the soft hadrons from the fluid freeze-out. We employ the new picture to investigate $\mathrm{Pb}-\mathrm{Pb}$ collisions at $2.76 \mathrm{TeV}$. We discuss the centrality and $p_{t}$ dependence of particle production and long range dihadron correlations at small and large $p_{t}$.
\end{abstract}

\section{INTRODUCTION}

Traditionally the physics of ultrarelativistc heavy ion collisions is discussed in terms of different categories like collective dynamics, parton-jet physics, and fluctuationcorrelation studies, although these different topics are highly correlated. In this article, a complete dynamical picture of particle production at all $p_{t}$ scales will be presented, which accounts for the production and evolution of bulk matter and jets, and the very important interaction between the two components (which is not only the well known parton energy loss). The consequences of these interactions can be nicely seen in long range dihadron correlations.

The physical picture of our approach is the following: Initial hard scatterings result in mainly longitudinal flux tubes, with transversely moving pieces carrying the $p_{t}$ of the partons from hard scatterings. These flux tubes constitute eventually both bulk matter (which thermalizes, flows, and finally hadronizes) and jets, according to some criteria based on partonic energy loss. We will consider a sharp fluid freeze-out hypersurface, defined by a constant temperature. Freeze-out here simply means the end of the fluid phase, but the hadrons still interact. High energy flux tube segments will leave the fluid, providing jet hadrons via the usual Schwinger mechanism of flux-tube breaking caused by quark-antiquark production.

But the jets may also be produced at the freeze-out surface. Here we assume that the quark-antiquark needed for the flux tube breaking is provided by the fluid, with properties (momentum, flavor) determined by the fluid rather than the Schwinger mechanism. Considering transverse fluid velocities up to $0.7 \mathrm{c}$, and thermal parton momentum distributions, one may get a "push" of a couple of $\mathrm{GeV}$ to be added to the transverse momentum of the string segment. This will be a crucial effect for intermediate $p_{t}$ jet hadrons.

There is another important issue. Even for hadrons with transverse momenta of $10-20 \mathrm{GeV}$, there is a large probability of a jet hadron formation before it enters the dense hadronic medium. This means a significant probability of scatterings between jet hadrons and soft hadrons (from freeze-out), having essentially two consequences: an increase of low $p_{t}$ particle production, and a reduction of yields at high $p_{t}$. In addition there are of course the well known hadronic interactions between the soft hadrons.

We have discussed different processes which all affect $p_{t}$ spectra. It is, however, possible to disentangle different contributions, by looking at dihadron correlations. These are extremely useful tools heavily used by experimental groups at the RHIC and the LHC [1 5$]$. Recently, the CMS and ALICE collaborations published results on such correlations in $\mathrm{Pb}-\mathrm{Pb}$ collisions at 2.76 $\mathrm{GeV}$ and different centralities, over a more or less broad range in relative pseudorapidity $(\Delta \eta)$ and full coverage of the relative azimuthal angle $(\Delta \phi)[4,5]$. Different combinations of transverse momenta $p_{t}^{\text {assoc }}$ and $p_{t}^{\text {trigg }}$ of associated and trigger particles in the range between 0.25 
$\mathrm{GeV} / \mathrm{c}$ and $15 \mathrm{GeV} / \mathrm{c}$ are investigated. Considering long range correlations $(|\Delta \eta|>A, A \geq 0.8)$, the coefficients $V_{n \Delta}$ of the harmonic decomposition factorize as $V_{n \Delta}=v\left(p_{t}^{\text {assoc }}\right) v\left(p_{t}^{\text {trigg }}\right)$ - not only for small transverse momenta but also for example for large $p_{t}^{\text {trigg }}$ and small $p_{t}^{\text {assoc }}$. For small momenta the situation seems to be clear: the correlation is flow dominated. But factorization does not necessarily mean that both hadrons carry the flow from the fluid! This can in particular not be the answer for observed correlations for large $p_{t}^{\text {trigg }}$ - here we have to deal with an interaction between the flowing bulk and jets, which makes the observed correlations very interesting in particular as a test of our ideas concerning bulk-jet separation and interaction.

Another challenge: The ATLAS collaboration showed recently results [ 6] on elliptical flow of charged particles with respect to an event plane in the opposite $\eta$ hemisphere (also a kind of long range correlation). The $v_{2}$ values are quite large up to values of $p_{t}=20 \mathrm{GeV} / \mathrm{c}$, for eight different centrality ranges. Can we understand this in a quantitative fashion?

The heavy ion results shown in this paper are based on 2000000 events simulated with EPOS2.17v3. A central $(0-5 \%) \mathrm{Pb}-\mathrm{Pb}$ event takes on the average around 2 HSO6 hours CPU time, using machines with an average scaling factor of 8.7 [7]. Always six events share the same parton configuration and hydrodynamic evolution, with only particle production and hadronic rescattering being redone (to gain statistics). This is taken care of when considering mixed events in correlation studies.

\section{THE BASIS: FLUX TUBES FROM A MULTIPLE SCATTERING APPROACH}

The starting point is a multiple scattering approach corresponding to a marriage of Gribov-Regge theory and perturbative QCD (pQCD), see Fig. 1. An elementary scattering corresponds to a parton ladder, containing a hard scattering calculable based on pQCD, including initial and final state radiation (for details see [8]). These ladders are identified with flux tubes (see Fig. 2), which are mainly longitudinal objects, with transversely moving parts, carrying the transverse momenta of the hard scatterings. These objects are also referred to as kinky strings. One should note that here multiple scattering does not mean just a rescattering of hard partons, it rather means a multiple exchange of complete parton ladders, leading to many flux tubes. In this case, the energy sharing between the different scatterings will be very important, to be discussed later.

The consistent quantum mechanical treatment of the multiple scattering is quite involved, it is based on cutting rule techniques to obtain partial cross sections, which are then simulated with the help of Markov chain techniques

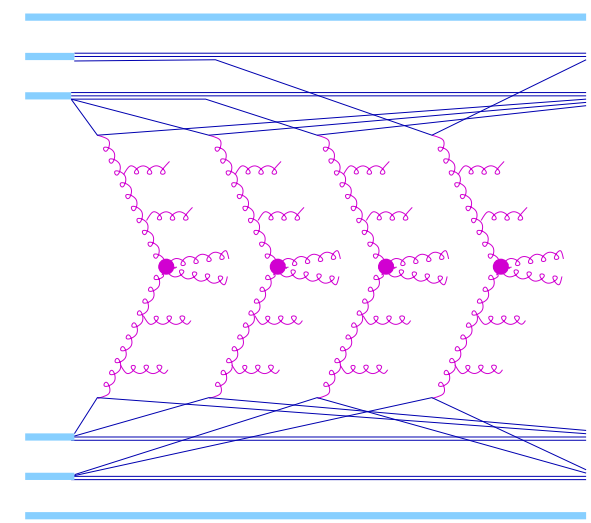

Figure 1: (Color online) Multiple scattering in nucleusnucleus collisions.

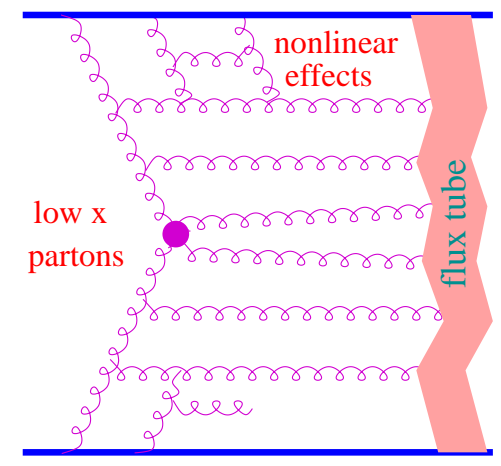

Figure 2: (Color online) An elementary parton ladder, whose final state is identified with a color flux tube (kinky string).

[9].

As said before, the final state partonic system corresponding to elementary parton ladders are identified with flux tubes. The relativistic string picture $10-12]$ is very attractive, because its dynamics is essentially derived from general principles as covariance and gauge invariance. The simplest possible string is a surface $X(\alpha, \beta)$ in $3+1$ dimensional space-time, with piecewise constant initial velocities $\partial X / \partial \beta$. These velocities are identified with parton velocities, which provides a one to one mapping from partons to strings. For details see [8, 9]. The high transverse momentum $\left(p_{t}\right)$ partons will show up as transversely moving string pieces, see Fig. 3(a). Despite the fact that in the $\mathrm{TeV}$ energy range most processes are hard, and despite the theoretical importance of very high $p_{t}$ partons, it should not be forgotten that the latter processes are rare, most kinks carry only few $\mathrm{GeV}$ of transverse momentum, and the energy is nevertheless essentially longitudinal. In case of elementary reactions, the strings will break (see Fig. 3(b) via the production of quark-antiquark pairs according to the so-called area law 
(a)

(b)
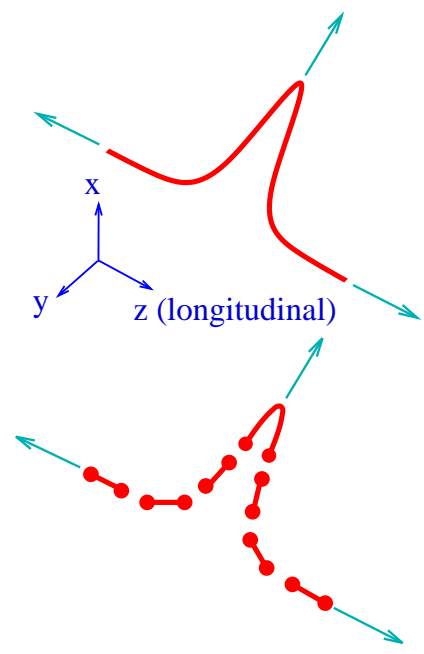

Figure 3: (Color online) (a) Flux tube with transversely moving part (kinky string) in space, at given proper time.

(b) Flux tube breaking via $q-\bar{q}$ production, which screens the color field (Schwinger mechanism).

[8, 9, 13, 14]. The string segments are identified with final hadrons and resonances.

This picture has been very successful to describe particle production in electron-positron annihilation or in proton-proton scattering at very high energies. In the latter case, not only low $p_{t}$ particles are described correctly, for example for pp scattering at $7 \mathrm{TeV}[15,16]$, also jet production is covered. As discussed earlier, the high transverse momenta of the hard partons show up as kinks, transversely moving string regions. After string breaking, the string pieces from these transversely moving areas represent the jets of particles associated with the hard partons. To demonstrate that this picture also works quantitatively, we compute the inclusive $p_{t}$ distri-

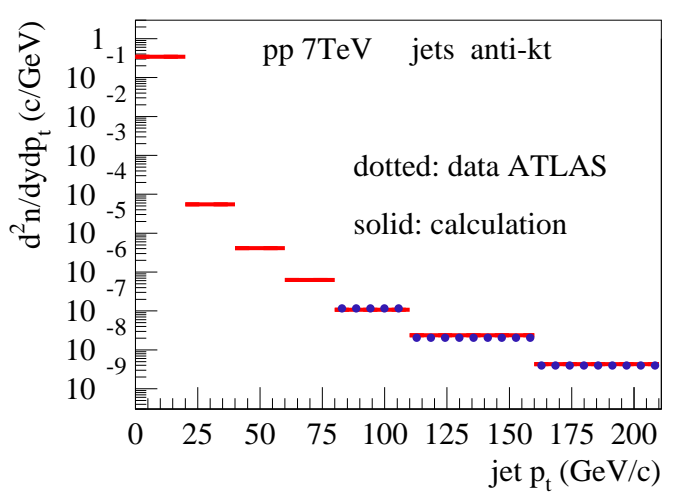

Figure 4: (Color online) Inclusive $p_{t}$ distribution of jets. We show the calculation (full lines) compared to ATLAS data 18] (dotted lines).

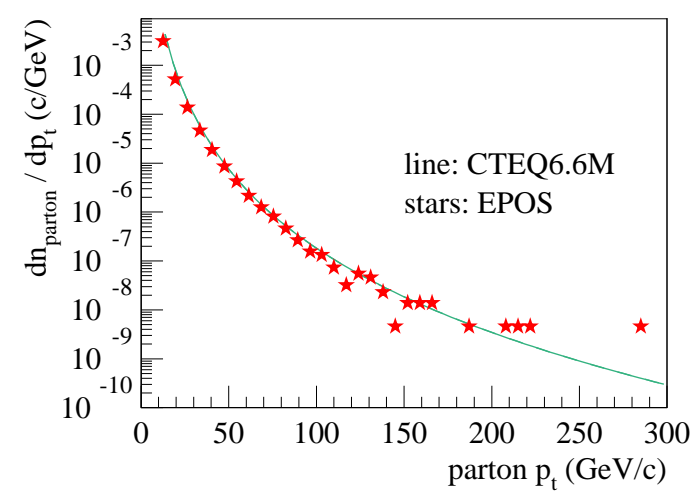

Figure 5: (Color online) Transverse momentum distribution of partons: We compare our results (red stars) with a parton model calculation (dotted line).

bution of jets, reconstructed with the anti-kt algorithm [17] and compare with data [18], see Fig. 4. In Fig. [5. we compare our $p_{t}$ distribution of partons with a parton model calculation based on CTEQ6 parton distribution functions [19], in both cases leading order with a K-factor of 2 .

\section{JET - BULK SEPARATION}

In heavy ion collisions and also in high multiplicity events in proton-proton scattering at very high energies, the density of strings will be so high that the strings cannot decay independently as described above. Here we have to modify the procedure as discussed in the following. The starting point are still the flux tubes (kinky strings) originating from elementary collisions. These flux tubes will constitute both, bulk matter which thermalizes and expands collectively, and jets. The criterion which decides whether a string piece ends up as bulk or jet, will be based on energy loss. In the following we consider a flux tube in matter, where "matter" first means the presence of a high density of other flux tubes, which then thermalize. A more quantitative discussion will follow.

Three possibilities should occur, referred to as A, B, C, see Fig. 6]:

A String segments far from the surface and/or being slow will simply constitute matter, they loose their character as individual strings. This matter will evolve hydrodynamically and finally hadronize ("soft hadrons").

B Some string pieces (like those close to transversely moving kinks) will be formed outside the matter, they will escape and constitute jets ("jet hadrons").

C There are finally also string pieces produced inside 
matter or at the surface, but having enough energy to escape and show up as jets ("jet hadrons"). They are affected by the flowing matter ("fluid-jet interaction").

Let us discuss how the above ideas are realized. In principle the formation and expansion of matter and the interaction of partons with matter is a dynamical process. However, the initial distribution of energy density and the knowledge of the initial momenta of partons (or string segments) allows already an estimate about the fate of the string segments. By "initial time" we mean some early proper time $\tau_{0}$ which is a parameter of the model. Strictly speaking, energy loss concerns partons, modifying eventually the kink momenta in our picture, and the momenta of the string segments after breaking will be reduced. We will therefore base our discussion on energy loss on string segments.

We estimate the energy loss $\Delta E$ of string segments along their trajectory to be

$$
\Delta E=k_{\text {Eloss }} E_{0} \int\left(\rho V_{0}\right)^{3 / 8} \max \left(1, \sqrt{E / E_{0}}\right) d L / L_{0}
$$

inspired by [20], where $\rho$ is the density of string segments at initial proper time $\tau_{0}, V_{0}$ is an elementary volume cell size (technical parameter, taken to be $0.147 \mathrm{fm}^{3}$ ), $L_{0}$ is a (technical) length scale (taken to be $1 \mathrm{fm}), E$ the energy of the segment in the "Bjorken frame" moving with a rapidity $y$ equal to the space-time rapidity $\eta_{s}, d L$ is a length element, and $k_{\text {Eloss }}$ and $E_{0}$ are parameters. We

(a)

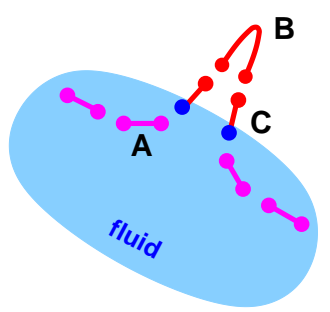

(b)

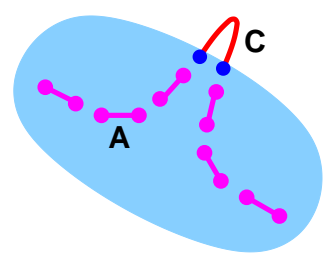

Figure 6: (Color online) Flux tube in matter (from other flux tubes, blue colored area). One distinguishes three types of behavior for string segments, noted as A, B, C (see text). The highest $p_{t}$ string segment may be (a) of type B or (b) of type C. introduce an energy cutoff $E_{0}$ to have sufficient energy loss for slowly moving segments.

A string segment will contribute to the bulk (type A segment), when its energy loss is bigger than its energy, i.e.

$$
\Delta E \geq E
$$

All the other segments are allowed to leave the bulk (type $\mathrm{B}$ or $\mathrm{C}$ segments). Only the bulk segments are used to determine the initial conditions for hydrodynamics, following the same procedure as explained in [8] (with some new elements, as discussed in the next section). Starting from this initial condition, the bulk matter will evolve according to the equations of ideal hydrodynamics till "hadronization", which occurs at some "hadronization temperature" $T_{H}$ [8]. Hadronization means that we change from matter description to particle description, but hadrons still interact among each other, realized via a hadronic cascade procedure [21], already discussed in [8].

After having performed the hydrodynamic expansion, we have to come back to the string segments which escape the bulk because their energy is bigger than the energy loss. We employ a formation time: the string segments are formed at times $t$ distributed as $\exp \left(-t / \gamma \tau_{\text {form }}\right)$, with some parameter $\tau_{\text {form }}$ which is taken to be $1 \mathrm{fm} / \mathrm{c}$. If the formation time is such that the segment is produced outside the "hadronization surface" defined by $T_{H}$, the segment will escape as it is (type B segment).

Most interesting are the segments which are formed inside but still escape, because they have $E>\Delta E$. These are type $\mathrm{C}$ segments. They escape, but their properties change. Actually such a segment leaves "matter" at the hadronization surface at a particular space-time point $x$, which is characterized by some collective flow velocity $\vec{v}(x)$. We assume that the string breaking in this case is modified such that the quark and antiquark (or diquark) necessary for the string breaking are taken from the flowing fluid rather than being produced via the Schwinger mechanism. So the new string segment is composed of a quark and antiquark (diquark) carrying the flow velocity, and the string piece in between, which has not been changed. This string piece may or may not carry large momentum, depending on whether it is close to a kink or not, the former possibility shown in Fig. 7

In any case, due to the fluid-jet interaction, the properties of this segment change drastically compared to the normal fragmentation:

- The quark and antiquark (or diquark ) from the fluid provide a push in the direction of the moving fluid.

- The quark (antiquark) flavors are determined from Bose-Einstein statistics, with more strangeness production compared to the Schwinger mechanism. 


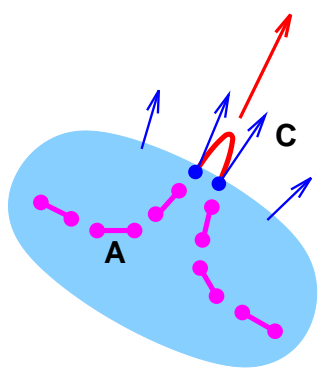

Figure 7: (Color online) A type $\mathrm{C}$ segment picks up quark and antiquark from the fluid, carrying momenta and flavor according to the fluid properties ("fluid-jet interaction").

- The probability $p_{\text {diq }}$ to have a diquark rather than an antiquark will be bigger compared to a highly suppressed diquark-antidiquark breakup in the Schwinger picture ( $p_{\text {diq }}$ is a parameter).

Our procedure has 4 parameters: $k_{\text {Eloss }}(=0.042), E_{0}$ $(=6 \mathrm{GeV}), \tau_{\text {form }}(=1 \mathrm{fm} / \mathrm{c}), p_{\text {diq }}(=0.22)$. It allows to cover in a single scheme the production of jets, of bulk, and the interaction between the two.

\section{FORMATION TIMES}

A crucial ingredient to the mechanism of fluid-jet interaction is the formation time of jet hadrons (the hadrons which leave the fluid). The probability distribution of the formation times $t$ of jet hadrons with gamma factors $\gamma$ is given as

$$
\text { prob } \sim \exp \left(-\frac{t}{\gamma \tau_{\text {form }}}\right),
$$

(we use $\tau_{\text {form }}=1 \mathrm{fm} / \mathrm{c}$ ). The probability of having a formation point inside the fluid is obtained as an integral over eq. (3),

$$
1-\exp \left(-\frac{t_{\max }}{\gamma \tau_{\text {form }}}\right)
$$

with $t_{\max }$ being the time corresponding to a formation at the fluid surface. Rather than making a simulation, we are going to present a very simple formula providing a rough estimate of the $p_{t}$ dependence of this probability. For a collision of two $\mathrm{Pb}$ nuclei in some centrality interval, characterized by the mean impact parameter $b$, we use $c t_{\max }=r_{\mathrm{Pb}}-b / 2$, where $r_{\mathrm{Pb}}$ is the radius parameter used in the Wood-Saxon distribution of nucleons. Considering transversely moving hadrons of mass $m$, we have $\gamma \approx$ $p_{t} / m c$. The estimate $P_{\text {inside }}$ of the probability to form (pre)hadrons inside the fluid is

$$
P_{\text {inside }}=1-\exp \left(-\frac{\left(r_{\mathrm{Pb}}-b / 2\right) m}{p_{t} \tau_{\text {form }}}\right) .
$$

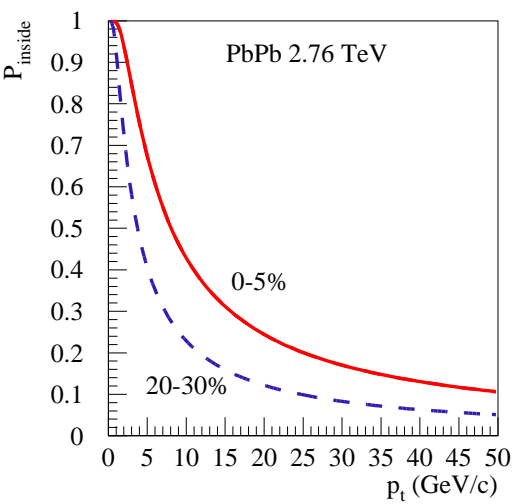

Figure 8: (Color online) The estimate $P_{\text {inside }}$ to form (pre)hadrons inside the fluid, as a function of $p_{t}$, for $\mathrm{Pb}-\mathrm{Pb}$ collisions at $2.76 \mathrm{TeV}$. We show the curves for the $0-5 \%$ and the $20-30 \%$ most central events.

In Fig. 8, we show the result for the $0-5 \%$ and the 20 $30 \%$ most central events in $\mathrm{Pb}-\mathrm{Pb}$ collisions at $2.76 \mathrm{TeV}$, using $c \tau_{\text {form }}=1 \mathrm{fm}, m c^{2}=1 \mathrm{GeV}, r_{\mathrm{Pb}}=6.5 \mathrm{fm}$, and for the average impact parameters $b=1.8 \mathrm{fm}(0-5 \%)$ and $b=7.8 \mathrm{fm}(20-30 \%)$.

By construction, the probability $P_{\text {fluid-jet }}$ of having a fluid-jet interaction is equal to the probability of forming (pre)hadrons inside the fluid, so its estimate is given by $P_{\text {inside }}$. From Fig. 8, we see that the probability is quite large for intermediate values of $p_{t}$, but even large values $(50 \mathrm{GeV} / \mathrm{c})$ are significantly affected. Whether the effect of the interaction can be seen in some observable is a different question and will be discussed later.

Several authors have already discussed about "inmedium hadronization", see for example Ref. [22], where one also finds an overview about earlier models on this subject.

\section{HYDRODYNAMICS}

The bulk matter extracted as described above provides the initial condition for a hydrodynamic evolution. As explained in [8], we compute the energy momentum tensor and the flavor flow vector at some position $x$ (at $\tau=\tau_{0}$ ) from the four-momenta of the bulk string segments. The time $\tau=\tau_{0}$ is as well taken to be the initial time for the hydrodynamic evolution. This seems to be a drastic simplification, the justification being as follows: we imagine to have a purely longitudinal scenario (descibed by flux tubes) till some proper time $\tau_{\text {flux }}<\tau_{0}$. During this stage there is practically no transverse expansion, and the energy per unit of space-time rapidity does not change. This property should not change drastically beyond $\tau_{\text {flux }}$, so we assume it will continue to hold during thermalization between $\tau_{\text {flux }}$ and $\tau_{0}$. So although we cannot say anything 


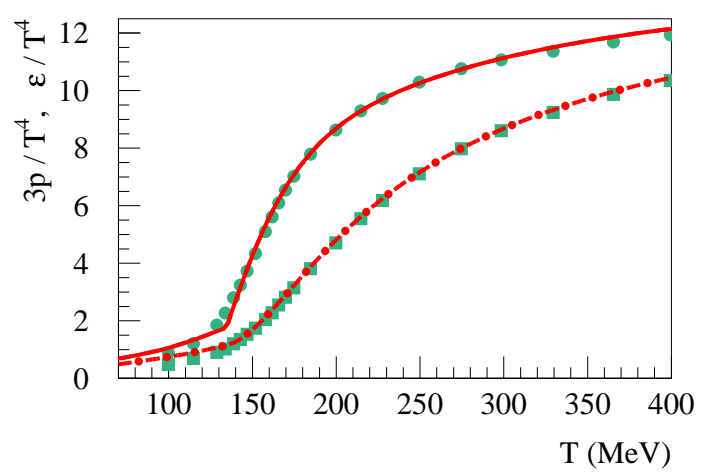

Figure 9: (Color online) Energy density and pressure versus temperature, for our equation-of-state (lines) compared to lattice data [23] (points).

about the precise mechanism which leads to thermalization, and therefore we cannot compute the real $T^{\mu \nu}$, we expect at least the elements $T^{00}$ and $T^{0 i}$ to stay close to the flux tube values, and we can use the flux tube results to compute the energy density. Only $T^{i j}$ will change considerably, but this does not affect our calculation much.

We employ three-dimensional ideal hydrodynamics as described in [8], with some modification to be discussed in the following. As in [8], we construct the equation of state as

$$
p=p_{Q}+\lambda\left(p_{H}-p_{Q}\right)
$$

where $p_{H}$ is the pressure of a resonance gas, and $p_{Q}$ the pressure of an ideal quark gluon plasma, including bag pressure. We use an updated $\lambda$ :

$$
\lambda=\exp \left(-z-3 z^{2}\right) \Theta\left(T-T_{c}\right)+\Theta\left(T_{c}-T\right),
$$

with

$$
z=x /(1+x / 0.77), \quad x=\left(T-T_{c}\right) / \delta,
$$

using $\delta=0.24 \exp \left(-\mu_{b}^{2} / 0.4^{2}\right)$. The new $\lambda$ provides an equation of state in agreement with recent lattice data [23], see Fig. 9],

Apart of the new equation of state, we use the same procedure to obtain energy density and pressure from the string segments, as described in [8]. However,

- doing the calculation for $\mathrm{Pb}-\mathrm{Pb}$ collisions at 2.76 $\mathrm{TeV}$, we get too much elliptical flow (20-30\%), a hint that one should include viscosity.

Taking the usual small radii of the elementary flux-tubes, we get extremely strongly fluctuating energy densities (in the transverse plane). Viscosity will quickly reduces these strong fluctuations.

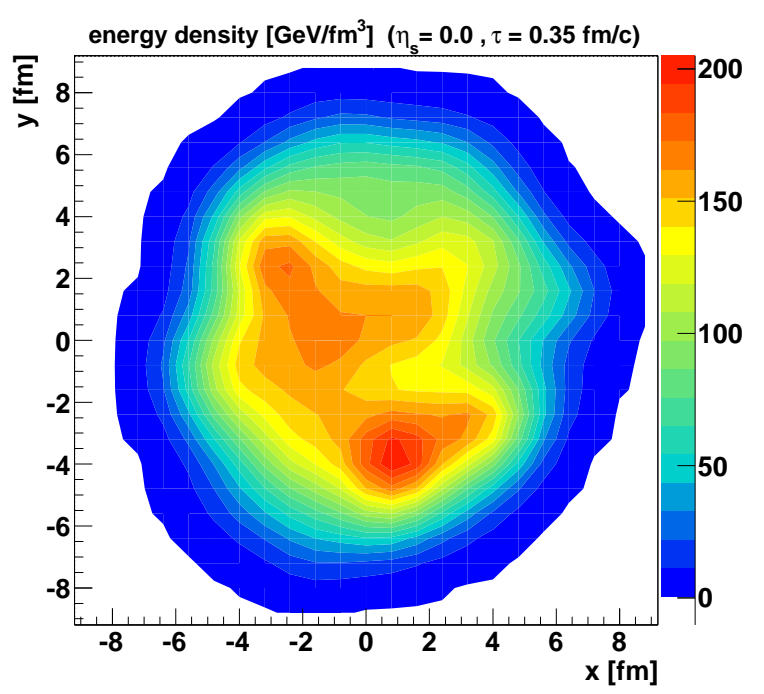

Figure 10: (Color online) Initial energy density in a central $\mathrm{Pb}-\mathrm{Pb}$ collision at $2.76 \mathrm{TeV}$, at a space-time rapidity $\eta_{s}=0$, as a function of the transverse coordinates $x$ and $\mathrm{y}$. We take artificially large values of the flux tube radii - which provides relatively smooth initial conditions - to mimic viscous effects.

- We try to mimic viscous effects by taking artificially large values of the flux tube radii (we take $1 \mathrm{fm}$ ), in order to get smoother initial conditions.

This has the effect of reducing the elliptical flow by 20$30 \%$, as needed. In Fig. 10, we show an example of such an initial energy density.

\section{CENTRALITY DEPENDENCE OF THE MULTIPLICITY AND FAKE SCALING LAWS}

As a very first check of the new approach, we consider the centrality dependence of the charged particle yield. Although very basic, there is quite some confusion about this quantity. Whereas hard processes scale roughly with the number of binary nucleon-nucleon collisions (in a simple geometrical picture), the centrality dependence of the charged particle yield (dominated by low $p_{t}$ ) is very different: it looks more like a scaling with the number of participating nucleons. This reminds us of the good old "wounded nucleon model", which has a physical meaning - at low energies: the projectile and target nucleons are excited ("wounded"), and this is the main source of particle production.

Amazingly, this approximate participant scaling holds also at higher energies, the centrality dependence at the LHC is almost identical to the one at the RHIC [24]. This is quite strange, since one might believe that at higher energies hard processes dominate, so one could expect more binary scaling. But this is not the case. 
What do we get in the multiple scattering approach? In Fig. 11, we plot the yield per participant

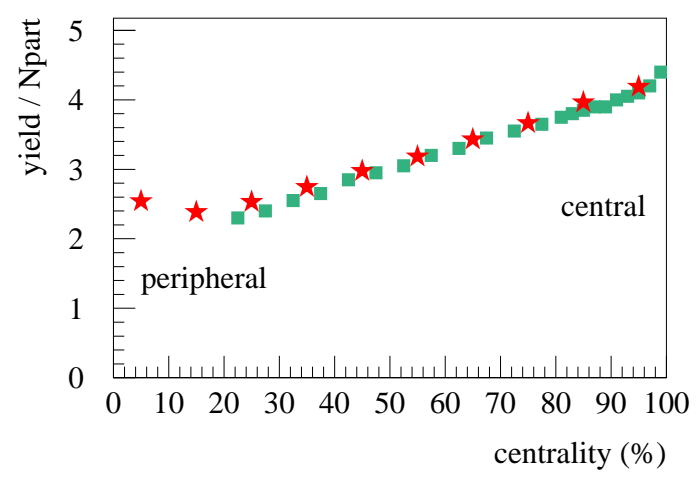

Figure 11: (Color online) Centrality dependence of the charged particle yield. Calculations (stars) are compared to ATLAS data [25] (circles).

$(d n / d \eta(0) /$ Npart ) as a function of the centrality. Npart is the number of participating nucleons. As in the data [25], we obtain a moderately increasing yield per participant. How can this happen? How can one get something like a wounded nucleon result at the LHC? In the model we can of course easily check the relative contribution of particle production from remnant decays. In Fig. 12, we

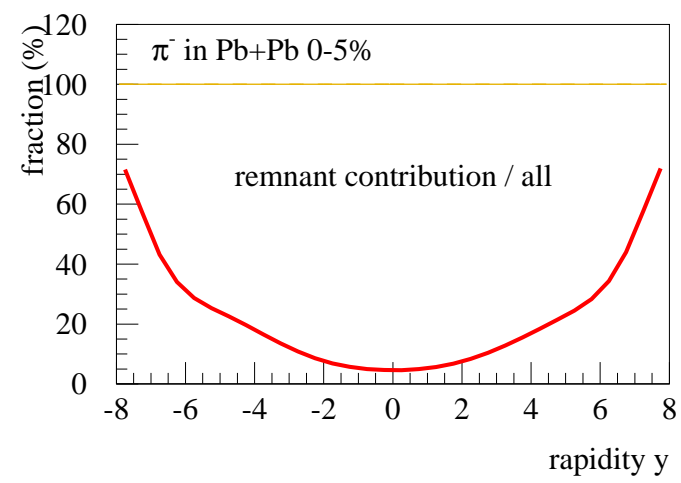

Figure 12: (Color online) Relative fraction of particle production from remnants versus rapidity. The contribution from remnants at mid-rapidity is very small.

plot the relative fraction of particle production from remnants as a function of the rapidity. As expected, remnant particle production is important at large rapidities, but the contribution at mid-rapidity is close to zero. So the physical mechanism of soft particle production is not a wounded nucleon picture.

In our approach, the source of particle production is the flux tubes, originating from elementary scatterings, which are in principle proportional to the number of binary nucleon-nucleon collisions. But there are impor-

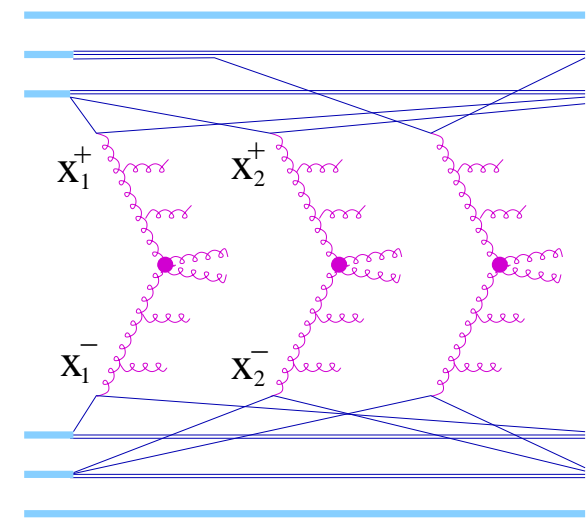

Figure 13: (Color online) Energy sharing in AA scatterings. The sum of all "ladder end" light cone momentum fractions $x_{k}^{ \pm}$linked to a given remnant and the remnant fraction $x_{\mathrm{remn} i}^{ \pm}$ have to add up to unity.

tant effect due to energy conservation and shadowing, discussed in detail in [8, 9]. In our multiple scattering approach (which determines the initial conditions), the complete AA scattering amplitude is expressed in terms of elementary contributions, which are parton ladders, later showing up as strings. Each parton ladder is characterized by the light cone momentum fractions $x_{k}^{+}$and $x_{k}^{-}$of the "ladder ends", which are the outer partons of the ladder, see Fig. 13 (also transverse momenta are considered, but not discussed here).

It is a unique feature of our approach that we do a precise bookkeeping of energy and momentum: For each nucleon (projectile or target) the initial energy-momentum has to be shared by all the ladders connected to this nucleon and the nucleon remnants, i.e. for all nucleons $i$ we have

$$
\sum_{\text {all ladders } k} x_{k}^{ \pm}+x_{\mathrm{remn} i}^{ \pm}=1
$$$$
\text { connected to nucleon } i
$$

where $x_{\mathrm{remn} i}^{ \pm}$is the momentum fraction of the nucleon remnant $i$. These are very strong conditions, which affect the results substantially, see [9].

The most important consequence relevant for our discussion here is the fact that parton ladders leading to low $p_{t}$ particles are suppressed compared to what is expected from binary scaling. We will get a nuclear modification factor which is less than one at low $p_{t}$, as shown in Fig. 14 for central $\mathrm{Pb}-\mathrm{Pb}$ collisions at $2.76 \mathrm{TeV}$. So although particle production at central rapidities in very high energy collisions is dominated by binary scattering (providing the initial energy density), particle production does not increase proportional to the number of binary scatterings, due to energy conservation.

It is absolutely necessary that binary scaling is broken 
K. Werner et al.

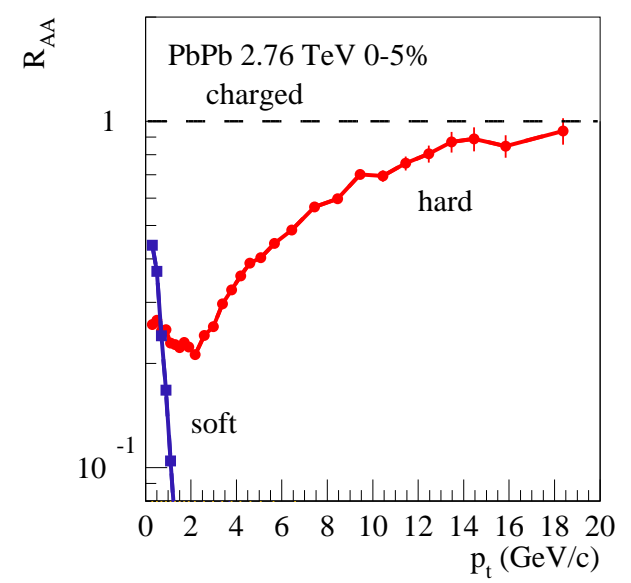

Figure 14: (Color online) Nuclear modification factor $R_{A A}=$ $N_{\text {coll }}^{-1}\left(d n_{A A} / d p_{t}\right) /\left(d n_{p p} / d p_{t}\right)$ vs transverse momentum $p_{t}$, showing the breaking of binary scaling at low $p_{t}$ (due to energy conservation). The resulting "approximate participant scaling at low $p_{t}$ " is a pure coincidence!

at low $p_{t}$, because it is simply an experimental fact. The usual explanation is a two component picture: hard scattering at high $p_{t}$ which shows binary scaling and a soft component which scales as the number of participants In our picture, binary collisions determine everything. But certain binary collisions are suppressed due to energy conservation, leading to a deviation from $R_{A A}=1$.

We will discuss the $p_{t}$ dependence of $R_{A A}$ in the next section. Here we present for completeness the pseudorapidity distributions of charged particles for different centralities, see Fig. 15, where we compare our calculation with data from ATLAS [25].

\section{TRANSVERSE MOMENTUM DEPENDENCE OF PARTICLE YIELDS: IMPORTANCE OF HADRONIC RESCATTERING OF SOFT AND JET HADRONS}

We first investigate particle production at low transverse momenta. In figs. 16, 17 and 18, we show transverse momentum distributions of pions, kaons, and protons, for central and semi-peripheral $\mathrm{Pb}-\mathrm{Pb}$ collisions at $2.76 \mathrm{TeV}$. We compare the full calculation including hydrodynamic evolution and hadronic final state cascade (solid lines) with the calculation without cascade (dashed lines) and with data from ALICE [26].

In order to understand the results, one has to recall that not only the "soft" particles produced from the fluid may interact, but also the jet-particles having enough energy to escape the fluid may interact with these soft particles. In particular intermediate $p_{t}$ jet particles are can-

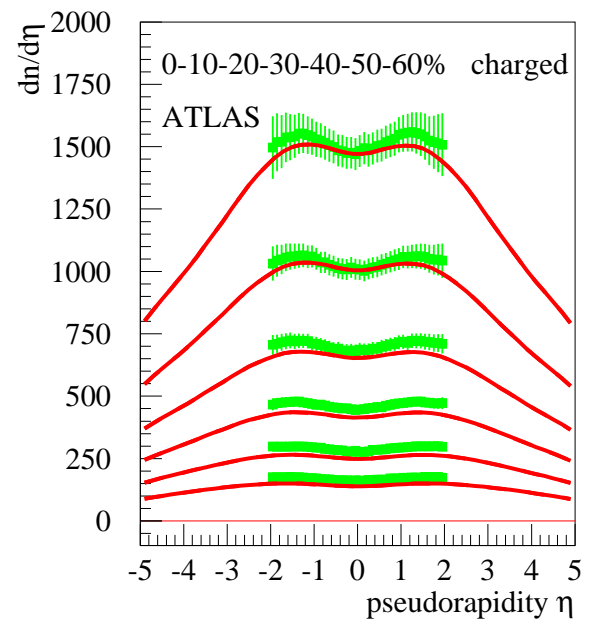

Figure 15: (Color online) Pseudorapidity distributions of charged particles for different centralities. The lines are calculations, the the circles are ATLAS data [25], see text.

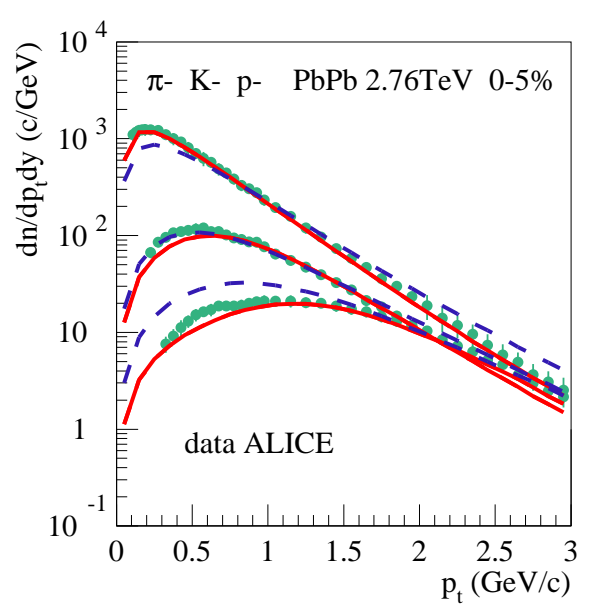

Figure 16: (Color online) Transverse momentum distributions of (from top to bottom) negative pion, kaons, and protons, in the $0-5 \%$ most central $\mathrm{Pb}-\mathrm{Pb}$ collisions at $2.76 \mathrm{TeV}$. We show the full calculation (solid lines) and the ones without hadronic cascade (dashed lines), compared to ALICE data (circles) [26].

didates, because their formation time will produce them just in the high density hadronic region. Let us discuss the consequences of these interactions, by comparing the solid and dashed curves in the figures.

- We see in particular in Fig. 16 a strong reduction of protons at low $p_{t}$ due to hadronic rescattering, which can be attributed to proton-antiproton annihilation among the soft hadrons.

- We see also a sizable increase of pion production at low $p_{t}$, which is due to inelastic rescatterings of jet 
Jets, Bulk Matter, and their Interaction

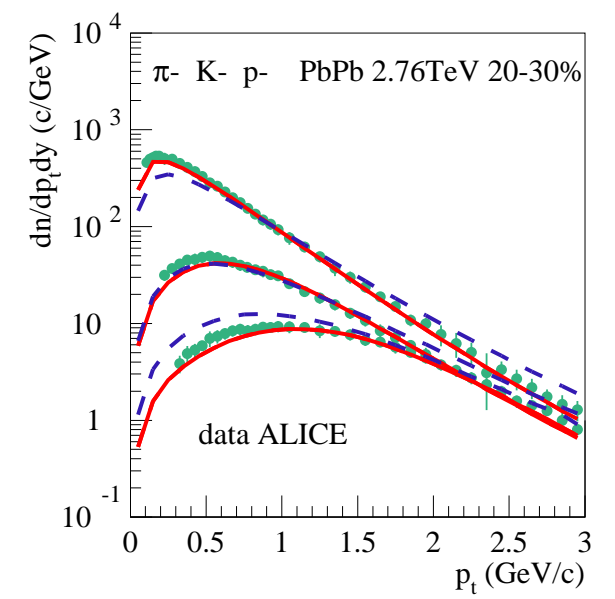

Figure 17: (Color online) Same as Fig. 16 but 20-30\% most central.

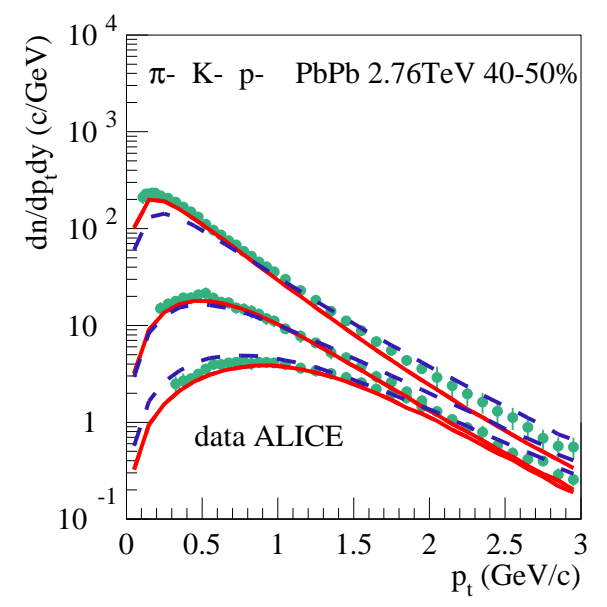

Figure 18: (Color online) Same as Fig. 16] but 40-50\% most central.

hadrons with soft ones

In figs. 16, 17, and 18, we only show results up to 3 $\mathrm{GeV} / \mathrm{c}$, because this is the range where data on protons, pions, and kaons are available. It is nevertheless interesting to know the effect of jet-soft scattering beyond 3 $\mathrm{GeV} / \mathrm{c}$. We therefore plot in Fig. 19 the ratio of the full calculation to the one without hadronic cascade, for the $p_{t}$ spectra of charged particles (dominated by pions) in central $\mathrm{Pb}-\mathrm{Pb}$ collisions at $2.76 \mathrm{TeV}$, up to $20 \mathrm{GeV} / \mathrm{c}$. There is a big effect at intermediate values of $p_{t}-$ up to $20 \mathrm{GeV} / \mathrm{c}$ ! In other words, jet-soft rescattering is very important in this range. Similar observation have already been made in [27] for $\mathrm{AuAu}$ collisions at the RHIC.

The big effect of the jet-soft interaction can be under-

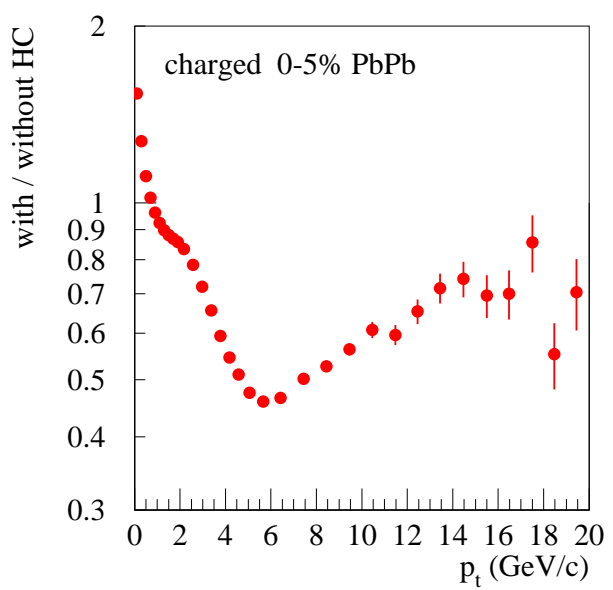

Figure 19: (Color online) Transverse momentum dependence of the ratio of the full calculation to the one without hadronic cascade, for charged particle production in the $0-5 \%$ most central $\mathrm{Pb}-\mathrm{Pb}$ collisions at $2.76 \mathrm{TeV}$.

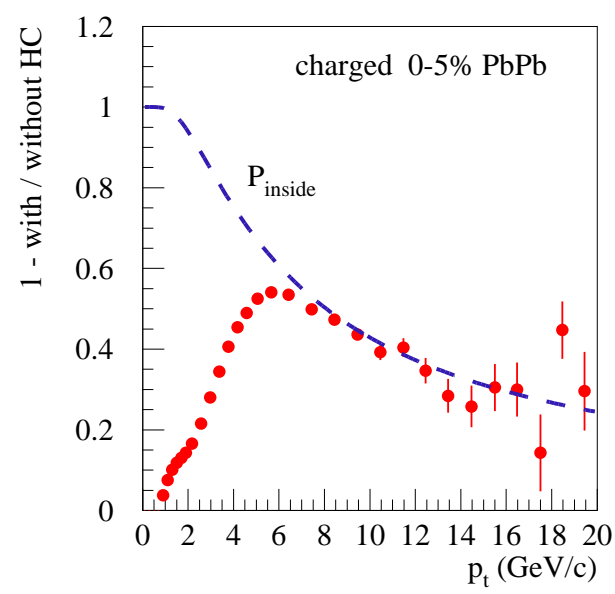

Figure 20: (Color online) Transverse momentum dependence of $1-R$ (red dots), with $R$ being the ratio plotted in Fig. 19 The dashed line is the estimate $P_{\text {inside }}$ of the probability to produce a jet hadron inside the fluid.

stood by plotting $1-R$, with $R$ being the ratio (with / without cascade) plotted in Fig. 19, together with the probability estimate $P_{\text {inside }}$ to produce a jet (pre)hadron inside the fluid, see fig. 20. These early produced hadrons go through the dense hadronic phase (of soft hadrons), and $P_{\text {inside }}$ is therefore also a measure of the probability of having a jet-soft interaction. We see indeed

$$
1-R=P_{\text {inside }},
$$

at large $p_{t}$ (in absolute terms, without adding factors). Even though we are running out of statistics, it is clear from the above discussion that the effect goes well beyond 


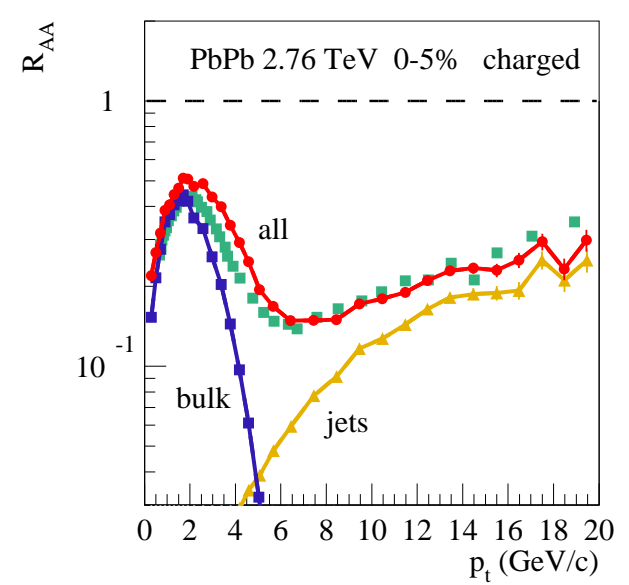

Figure 21: (Color online) The nuclear modification factor in $\mathrm{Pb}-\mathrm{Pb}$ at $2.76 \mathrm{TeV}$ vs $p_{t}$ : We compare data [28] (squares) with the full calculation (red line + circles) and its jet contribution (yellow line + triangles), as well as the bulk (hydro) contribution of a calculation without hadronic cascade (blue line + squares).

\section{$20 \mathrm{GeV} / \mathrm{c}$.}

To compare the $p_{t}$ spectra with experimental data, one uses often the so-called nuclear modification factor $R_{A A}$, which is the ratio of the inclusive transverse momentum spectrum of particles in nucleus-nucleus scatterings over the proton-proton ones, normalized by the number $N_{\text {coll }}$ of binary collisions. Doing this procedure, we obtain the curves shown in Fig. 21, where we plot our simulation results for charged particle production together with the data from ALICE [28]. We show the full model, including hydrodynamic evolution and final state hadronic cascade 21] and its jet contribution from the string segments which escaped from the bulk and which did not rescatter. We also show the bulk contribution (originating from the hadronized fluid) from the calculation without final state hadronic cascade. The two latter curves do not add up to give the full result - the difference is due to the "secondary interactions" discussed earlier:

- Fluid-jet interaction - pushing the jet hadrons at intermediate $p_{t}$ to higher transverse momenta.

- Jet-soft interactions between jet hadrons and soft ones from fluid freeze-out.

There are also soft-soft interactions (among soft hadrons from fluid freeze-out), which are important for baryon yields, but not so much for the charged particle results.

From the above discussion it is clear that even considering elementary quantities as charged particle yields, it is difficult to make any quantitative analysis without

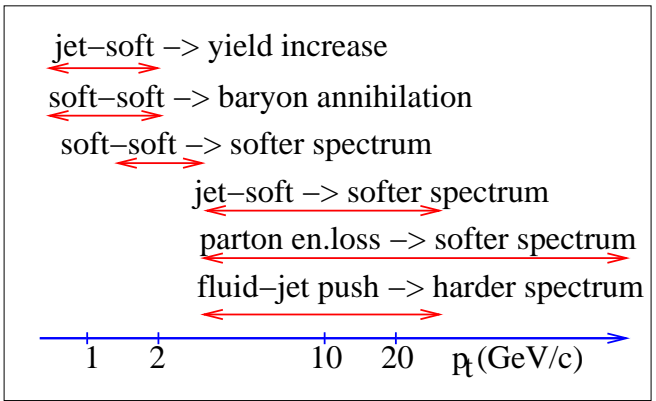

Figure 22: (Color online) Secondary interactions. The red arrows indicate the $p_{t}$ range which are affected.

considering these "secondary interactions". We sketch the different interactions in Fig. 22 .

\section{DIHADRON CORRELATIONS IN PB-PB AT 2.76 TEV}

Our prescription for bulk-jet separation and interaction should also strongly affect dihadron correlations, which provide much more information than simple spectra. With all parameters $\left(k_{\text {Eloss }}, E_{0}, \tau_{\text {seg }}, p_{\text {diq }}\right)$ being fixed from the considerations in the last section, we now compute dihadron correlation functions defined as

$$
R(\Delta \eta, \Delta \phi)=\frac{M}{S} \times \frac{S(\Delta \eta, \Delta \phi)}{M(\Delta \eta, \Delta \phi)}
$$

where $S$ is the number of pairs in real events, and $M$ the number of pairs for mixed events.

As an example, we show in Fig. 23 the correlation function for the $p_{t}$ of the trigger particle $\left(p_{t}^{\text {trigg }}\right)$ in the interval 4.5-5.5 GeV/c and the $p_{t}$ of the associated particle $\left(p_{t}^{\text {assoc }}\right)$ in the range $2-2.5 \mathrm{GeV} / \mathrm{c}$, in the $0-10 \%$ most central $\mathrm{Pb}-\mathrm{Pb}$ collisions at $2.76 \mathrm{TeV}$. Besides the jet peak at $\Delta \phi=0$ and $\Delta \eta=0$, we clearly identify a completely flat ridge over the full range in $\Delta \eta$ at $\Delta \phi=0$.

The reason for the ridge structure is the fact that there is an azimuthal asymmetry of the initial energy density (see Fig. 10). Although the energy density is biggest around space-time rapidity $\eta_{s}=0$ and drops fast towards forward and backward $\eta_{s}$, the shape of the asymmetry is preserved. This leads finally to an asymmetric flow, again very similar at different values of $\eta_{s}$, and this "makes" the long range correlation at $\Delta \phi=0$.

The smooth $\eta_{s}$ dependence of the energy density in our approach (see Fig. 24) is due to the fact the energy density is calculated from flux tubes. And these flux tubes have to be treated correctly as continuous longitudinal objects (as we do). In an earlier version, we treated flux tubes via the randomly (in $\eta_{s}$ ) distributed flux tube segments, obtained from a string fragmentation procedures. 
This gives a bumpy structure in $\eta_{s^{-}}$the ridge is not flat any more but has a Gaussian shape! So the flux-tube basis is an essential ingredient for obtaining a perfect ridge shape, as observed in the data.

In Fig. 25, we show a correlation function for $p_{t}^{\text {trigg }}$ in the interval 5.5-8.0 GeV/c and $p_{t}^{\text {assoc }}$ in the range 2-2.5 $\mathrm{GeV} / \mathrm{c}$, again in the $0-10 \%$ most central $\mathrm{Pb}-\mathrm{Pb}$ collisions at $2.76 \mathrm{TeV}$. Although the trigger $p_{t}$ is too large to originate from freeze-out (from the flowing fluid), one still observes a ridge structure, which is due to the fluid-jet interaction. Let us consider again the situation of an initial azimuthal anisotropy in the energy density which is transported into a corresponding anisotropy in the flow, as discussed earlier. We sketch in Fig. 26 the (somewhat exaggerated) situation of a triangular transverse flow pattern with maximal flow around $\phi=0^{\circ}, 120^{\circ}$, and $240^{\circ}$ (with respect to the $y$-axis). The flow maxima are indicated by blue arrows. Again it is very important that this flow pattern is (not necessarily in magnitude, but in shape) very similar at different longitudinal positions - in the figure indicated by the two transverse planes $\mathbf{P}$ and $\mathbf{P}^{\prime}$, corresponding to two different space-time rapidities $\eta_{s}$ and $\eta_{s}^{\prime}$. A soft hadron (S) produced at $\eta_{s}$ at the fluid surface close to the position of maximal flow (for example at $\phi=0^{\circ}$ ), will be boosted by the latter one and therefore carry information about this flow. A jet hadron $(\mathbf{J})$ produced at $\eta_{s}^{\prime}$ at the same angle $\left(\phi=0^{\circ}\right)$ close to the surface, will pick up a quark and an antiquark, both carrying flow, which adds the corresponding transverse momentum to the $p_{t}$ of the string segment (red element in the figure). It is the same flow which affects the jet hadron at $\eta_{s}^{\prime}$ and the soft hadron at $\eta_{s}$, which creates the

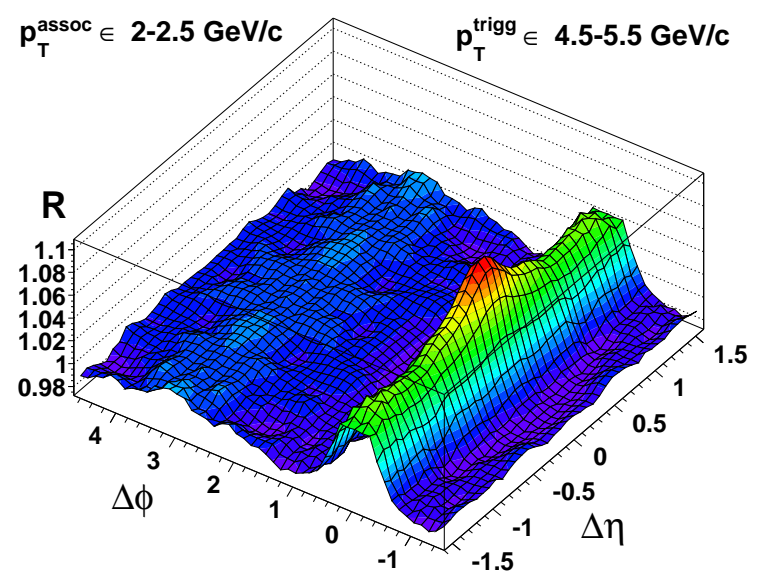

Figure 23: (Color online) Calculated dihadron correlation function for $p_{t}^{\text {trigg }}$ in the interval $4.5-5.5 \mathrm{GeV} / \mathrm{c}$ and $p_{t}^{\text {assoc }}$ in the range $2-2.5 \mathrm{GeV} / \mathrm{c}$ in the $0-10 \%$ most central $\mathrm{Pb}-\mathrm{Pb}$ collisions at $2.76 \mathrm{TeV}$.

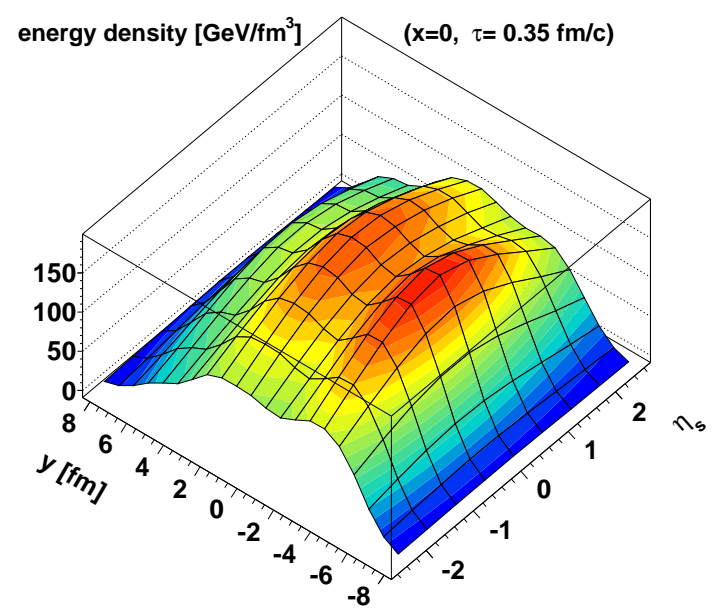

Figure 24: (Color online) The energy density for a single event in a central $\mathrm{Pb}-\mathrm{Pb}$ collisions at $2.76 \mathrm{TeV}$ as a function of the longitudinal variable $\eta_{s}$ and the transverse one $y$ (and for $x=0$ ).

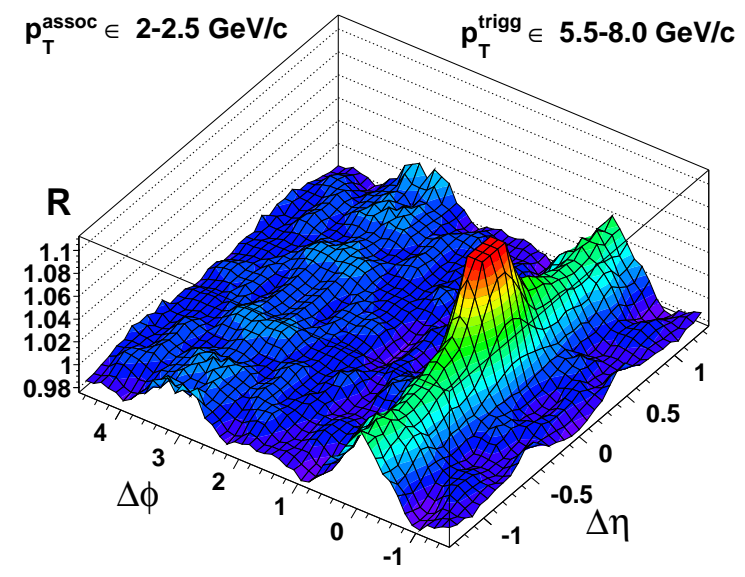

Figure 25: (Color online) Calculated dihadron correlation function for $p_{t}^{\text {trigg }}$ in the interval $5.5-8.0 \mathrm{GeV} / \mathrm{c}$ and $p_{t}^{\text {assoc }}$ in the range $2-2.5 \mathrm{GeV} / \mathrm{c}$ in the $0-10 \%$ most central $\mathrm{Pb}-\mathrm{Pb}$ collisions at $2.76 \mathrm{TeV}$.

dihadron correlation at $\Delta \phi=0$, the "ridge". The correlation remains visible, even when the flow contribution to the jet hadron is only $10 \%$, this is why the correlation is still present even for trigger transverse momenta beyond $10 \mathrm{GeV} / \mathrm{c}$.

We will now discuss some examples of semi-peripheral $\mathrm{Pb}-\mathrm{Pb}$ collisions at $2.76 \mathrm{GeV} / \mathrm{c}$. In Fig. 27, we show the correlation function for $p_{t}^{\text {trigg }}$ in the interval 3.5- 

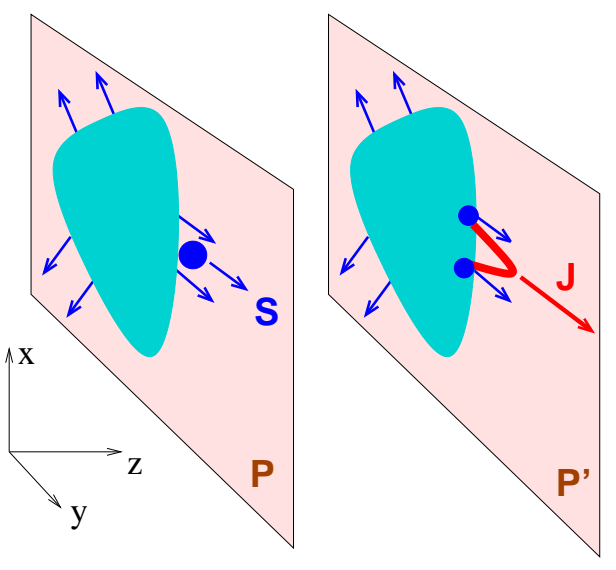

Figure 26: (Color online) Sketch of two cuts of the fluid volume corresponding to the space-time rapidities $\eta_{s}$ and $\eta_{s}^{\prime}$, the two corresponding transverse planes being $\mathbf{P}$ and $\mathbf{P}^{\prime}$. We show the example of a triangular flow pattern - the same at $\eta_{s}$ and $\eta_{s}^{\prime}$.

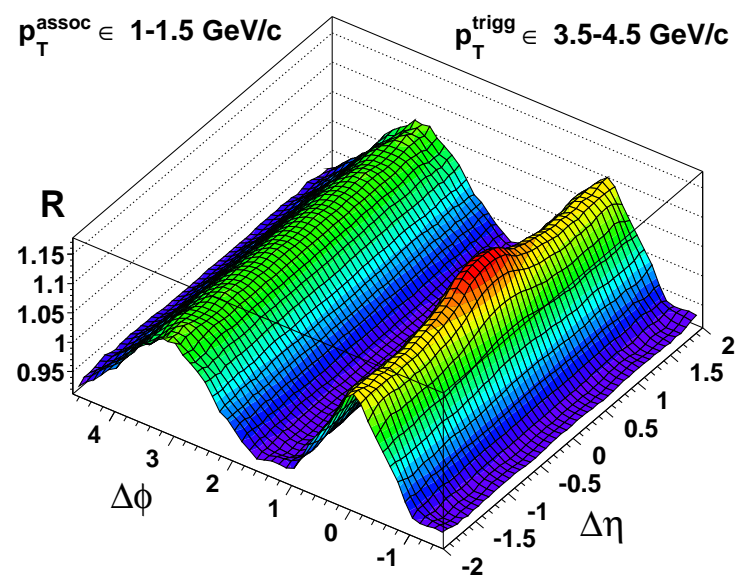

Figure 27: (Color online) Calculated dihadron correlation function for $p_{t}^{\text {trigg }}$ in the interval $3.5-4.5 \mathrm{GeV} / \mathrm{c}$ and $p_{t}^{\text {assoc }}$ in the range $1-1.5 \mathrm{GeV} / \mathrm{c}$ in the $40-50 \%$ most central $\mathrm{Pb}-\mathrm{Pb}$ collisions at $2.76 \mathrm{TeV}$.

$4.5 \mathrm{GeV} / \mathrm{c}$ and $p_{t}^{\text {assoc }}$ in the range $1-1.5 \mathrm{GeV} / \mathrm{c}$, in the $40-50 \%$ most central $\mathrm{Pb}-\mathrm{Pb}$ collisions at $2.76 \mathrm{TeV}$. It can be clearly seen from the figure that the elliptical flow $(\sim \cos (2 \Delta \phi))$ is dominant, besides the jet peak at $\Delta \eta=0, \Delta \phi=0$. But also here higher order harmonics $(\sim \cos (i \Delta \phi))$ contribute, as we will discuss later. In Fig. 28, we show another example of a correlation function for semi-peripheral collisions, with somewhat bigger trigger $p_{t}$. We use $p_{t}^{\text {trigg }}$ in the interval $5.5-8.0 \mathrm{GeV} / \mathrm{c}$ and $p_{t}^{\text {assoc }}$ in the range $2-2.5 \mathrm{GeV} / \mathrm{c}$. The $\Delta \eta$ range is chosen smaller to avoid to big statistical fluctuations. The jet contribution becomes dominant in this case, but when we cut off

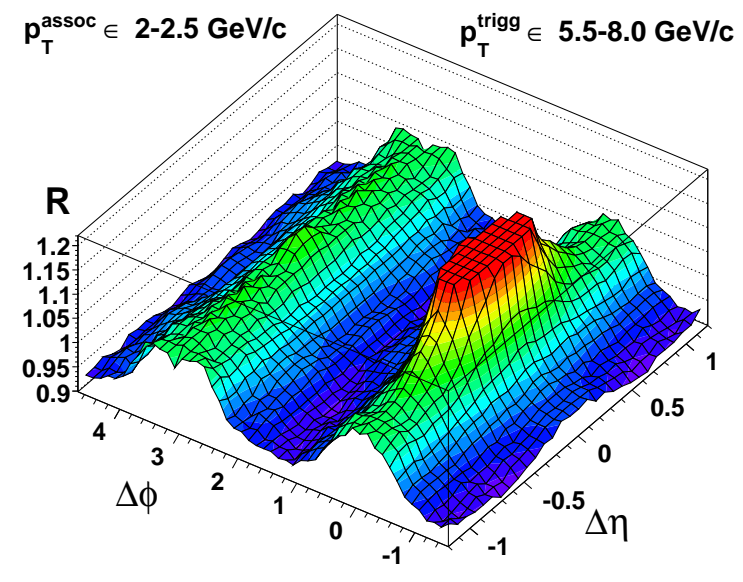

Figure 28: (Color online) Calculated dihadron correlation function for $p_{t}^{\text {trigg }}$ in the interval 5.5-8.0 GeV/c and $p_{t}^{\text {assoc }}$ in the range $2-2.5 \mathrm{GeV} / \mathrm{c}$ in the $40-50 \%$ most central $\mathrm{Pb}-\mathrm{Pb}$ collisions at $2.76 \mathrm{TeV}$. The jet peak has been cut for better visibility.

the jet peak, we clearly see a very similar elliptical flow structure as in the previous example.

The correlation functions are essentially flat as a function of $\Delta \eta$, for large $\Delta \eta$. One therefore gets complete information about the long range correlations by integrating over $\Delta \eta$,

$$
R(\Delta \phi)=\frac{1}{2(B-A)} \int_{A<|\Delta \eta|<B} R(\Delta \eta, \Delta \phi) d \Delta \eta,
$$

where we use $A=0.8$ and the maximum $B=2$. This function agrees perfectly with its Fourier decomposition,

$$
R(\Delta \phi)=1+\sum_{n=1}^{5} 2 V_{n \Delta} \cos (n \Delta \phi),
$$

using the first five terms. This is very convenient, because it allows to discuss the features of the correlation functions for different options for $p_{t}^{\text {trigg }}$ and $p_{t}^{\text {assoc }}$ by simply considering the Fourier coefficients.

In figs. 29 and 30, we plot some coefficients $V_{n \Delta}$ as a function of $p_{t}^{\text {trigg }}$ for different intervals of $p_{t}^{\text {assoc. The }}$ value of $p_{t}^{\text {trigg }}$ is actually the mean value in a certain interval, the largest interval being $8-15 \mathrm{GeV} / \mathrm{c}$. We compare our simulation (stars) with the results from ALICE [5] (circles).

In the semi-peripheral collisions of Fig. 29, we see clearly the dominance of elliptical flow: the $n=2$ coefficients are by far the largest. Nevertheless, also the higher harmonics contribute. We see in all cases an increase of the coefficients with $p_{t}^{\text {assoc }}$ and with $p_{t}^{\text {trigg }}$ up to 
(a)

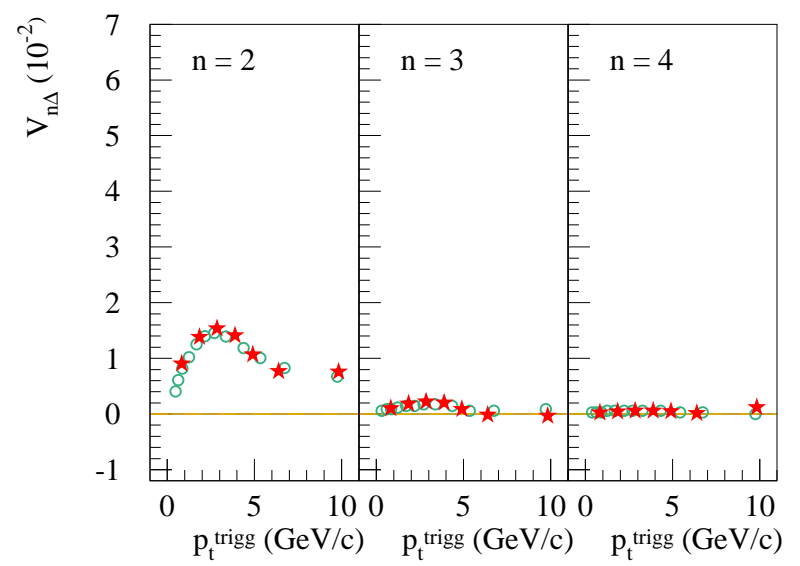

(b)

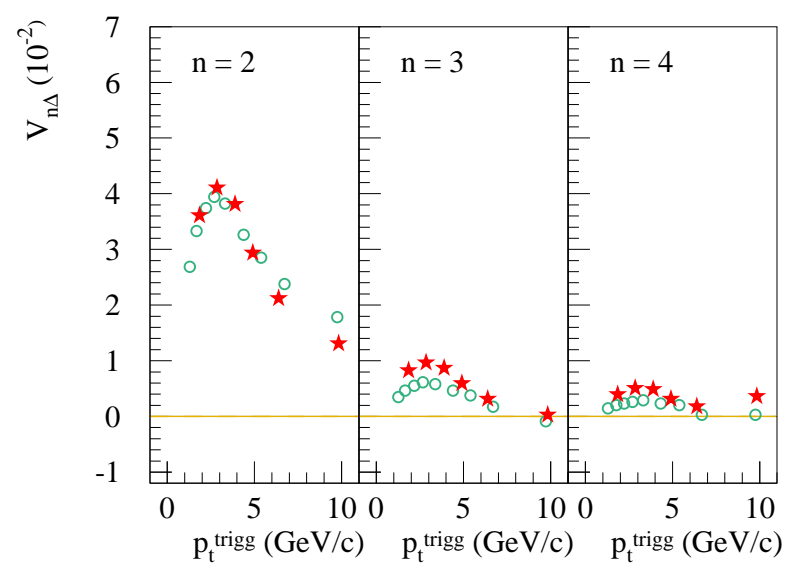

(c)

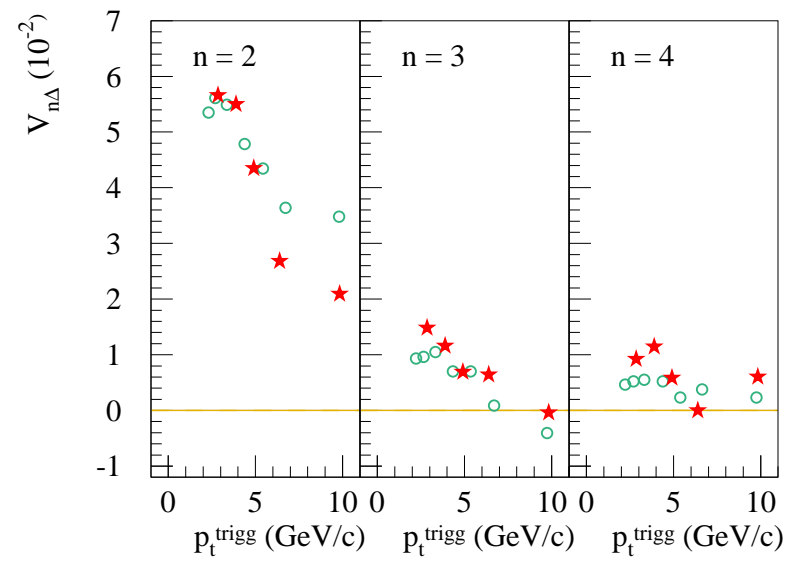

Figure 29: (Color online) The Fourier coefficients $V_{n \Delta}$ as a function of $p_{t}^{\text {trigg }}$ for $p_{t}^{\text {assoc }}$ within $0.25-0.5 \mathrm{GeV} / \mathrm{c}$ (a), 1$1.5 \mathrm{GeV} / \mathrm{c}(\mathrm{b}), 2-2.5 \mathrm{GeV} / \mathrm{c}$ (c), in the $40-50 \%$ most central $\mathrm{Pb}-\mathrm{Pb}$ collisions at $2.76 \mathrm{TeV}$. We compare the ALICE data [5] (circles) with calculations (red stars) .

values of around $2-3 \mathrm{GeV} / \mathrm{c}$. At the latter values the hydrodynamic flow contributes the most to the correlation between soft hadrons from the fluid.

For higher transverse momenta, the coefficients get smaller, because the correlation between soft particles (a)

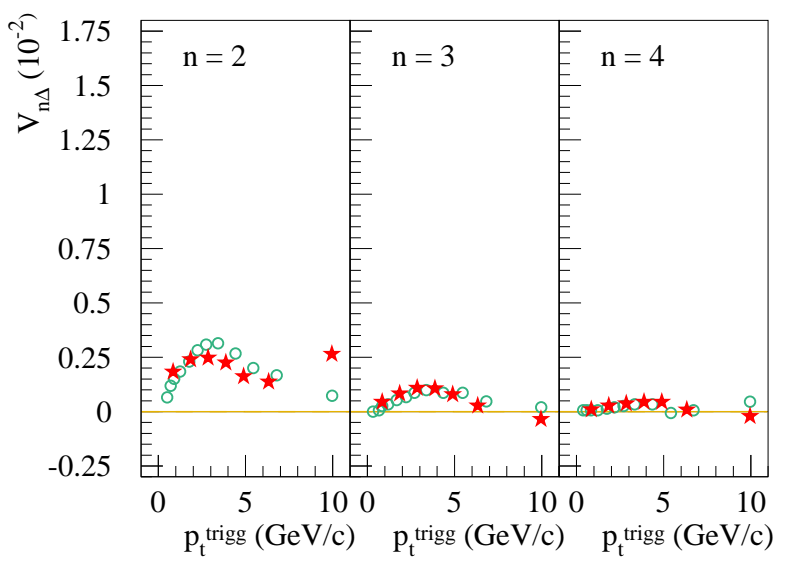

(b)

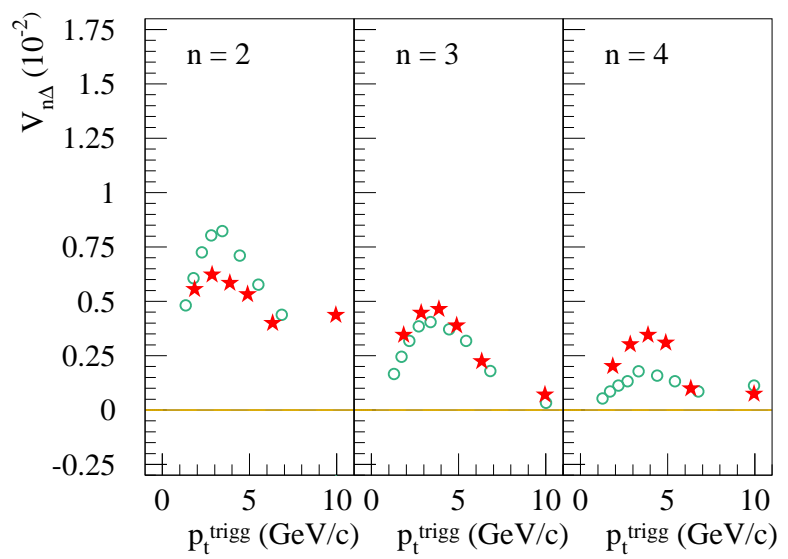

(c)

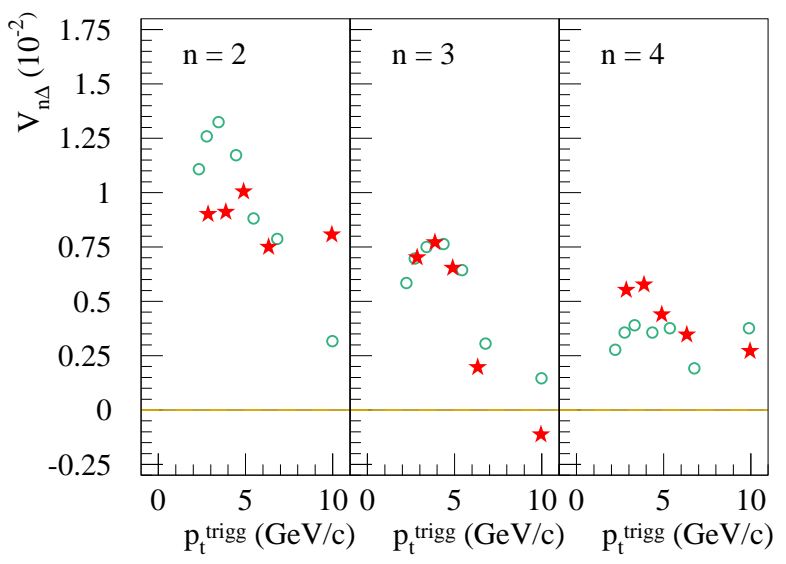

Figure 30: (Color online) Same as Fig. 29, but for the $0-10 \%$ most central $\mathrm{Pb}-\mathrm{Pb}$ collisions at $2.76 \mathrm{TeV}$. We compare the ALICE data [5] (circles) with calculations (red stars) .

dies out. But $V_{2 \Delta}$ does not at all drop to zero at high $p_{t}$ because here the correlations between soft and jet particles come into play - the jet particles which suffered a push by the fluid, as discussed earlier (fluid-jet interaction). The fluid transfers at maximum few $\mathrm{GeV} / \mathrm{c}$ of transverse momentum to the jet, but this is easily visible in the correlation (even at $20 \mathrm{GeV} / \mathrm{c}$ ). 


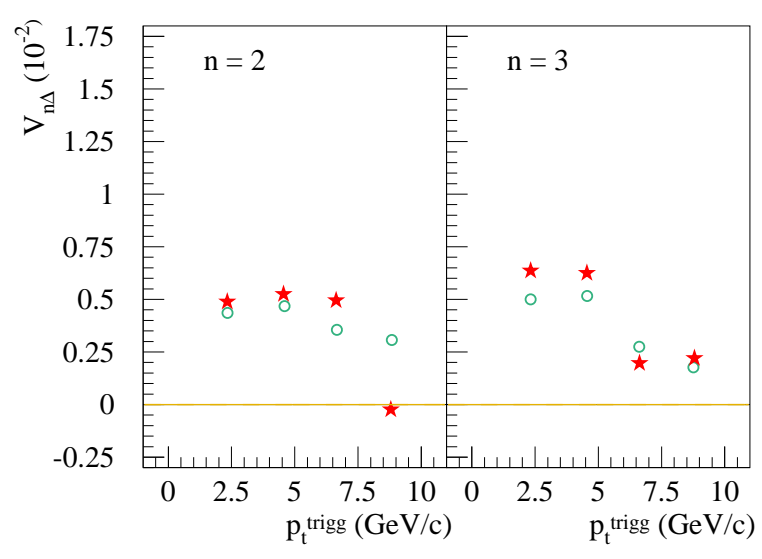

Figure 31: (Color online) The Fourier coefficients $V_{n \Delta}$ as a function of $p_{t}^{\text {trigg }}$ for $p_{t}^{\text {assoc }}$ within $2-4 \mathrm{GeV} / \mathrm{c}$ in the $0-5 \%$ most central $\mathrm{Pb}-\mathrm{Pb}$ collisions at $2.76 \mathrm{TeV}$. We compare the data [4] (squares) with calculations (red stars).

The results for semi-peripheral collisions are very robust and depend little on model parameters. The most important ingredient is the elliptical initial shape on the energy density, given by the nuclear geometry. The effects depend of course on the flow velocity at freeze-out, but this is not a parameter but itself a robust result (with a maximum around $0.7 \mathrm{c}$ ). Finally the results depend on the jet formation time, which should be around $1 \mathrm{fm} / \mathrm{c}$ (the value we actually took without really attempting a fine-tuning).

The $V_{2 \Delta}$ coefficients for central $\mathrm{Pb}-\mathrm{Pb}$ collisions (Fig. 30) are first of all smaller as compared to the semiperipheral ones (note the different scales in figs. 29 and 30), simply because the dominant effect of large initial ellipticity from the geometry is absent. Here the ellipticity is purely random. Apart from this, we observe the same features as for the semi-peripheral collisions: increase with transverse momenta up to $2-3 \mathrm{GeV} / \mathrm{c}$, then decrease. A big difference in central $\mathrm{Pb}-\mathrm{Pb}$ collisions compared to semi-peripheral ones is the fact that the higher harmonics and in particular $V_{3 \Delta}$ contribute substantially, because here both elliptical and triangular initial shape are of random origin (and therefore comparable), whereas for more peripheral collisions the geometrical elliptical shape dominates everything else.

In Fig. 30] it seems that our calculation underestimates $V_{2 \Delta}$, in particular for the largest $p_{t}^{\text {assoc }}$ range $(2-2.5$. GeV). Fortunately, similar data exist from CMS [4], for $p_{t}^{\text {assoc }}$ in the range $2-4 \mathrm{GeV} / \mathrm{c}$ in the $0-5 \%$ most central $\mathrm{Pb}-\mathrm{Pb}$ collisions. In Fig. 31, we plot the corresponding coefficients $V_{2 \Delta}$ and $V_{3 \Delta}$. Here we slightly overpredict the data!

\section{V2 AND FORMATION TIMES}

Whereas dihadron correlations provide the most complete information about particle production - in particular concerning the role of the "flowing" fluid, one may get the essential information by considering the elliptical flow coefficient $v_{2}$ of single particle production, which is defined as

$$
v_{2}=\left\langle\cos \left[2\left(\phi-\phi_{\text {Ref }}\right)\right]\right\rangle,
$$

where $\phi$ is the azimuth angle of a particle, and $\phi_{\text {Ref }}$ some reference plane. In [6], for the particles in the forward (backward) $\eta$ hemisphere, the reference plane is the event plane angle $\phi^{\text {backward }}\left(\phi^{\text {forward }}\right)$, obtained from counting all particles in the opposite hemisphere. The angles are obtained from

$$
\phi^{\text {backward/forward }}=\frac{1}{2} \tan ^{-1} \frac{\langle\sin 2 \phi\rangle}{\langle\cos 2 \phi\rangle},
$$

where the average is done in the forward / backward $\eta$ hemisphere within $3.2<|\eta|<4$.8. The $v_{2}$ coefficient is then computed as

$$
v_{2}=\left\langle\cos \left[2\left(\phi-\phi^{\text {forward } / \text { backward }}\right)\right]\right\rangle .
$$

Resolution correction is taken care of by dividing this expression by $R=\sqrt{\langle\cos [2 \Delta \phi]\rangle}$, with $\Delta \phi=\phi^{\text {backward }}-$ $\phi^{\text {forward }}$, as in ref [ $[$ ] $]$. Relating particles with the event plane of the opposite hemisphere, we have kind of a long range correlation, but less clean than using dihadron correlations with a $\Delta \eta>A$ requirement. But as mentioned before, the essential features can be seen as well. In Fig. 32. we plot $v_{2}$ as a function of the transverse momentum for different centralities in $\mathrm{Pb}-\mathrm{Pb}$ collisions at $2.76 \mathrm{TeV}$. The magnitude of the elliptical flow coefficients increase at low $p_{t}$ to reach a maximum around $2-3.5 \mathrm{GeV} / \mathrm{c}$ and then drop slowly at large $p_{t}$.

The behavior at high $p_{t}$ is the most interesting aspect: even at $10 \mathrm{GeV} / \mathrm{c}$, there is a significant amount of elliptical flow, due to the fluid-jet interaction, which pushes jet particles in the direction of the collective flow at the freeze-out surface (and this effect will continue up to even higher $p_{t}$, but we are simply running out of statistics). The high $p_{t}$ behavior is closely related to the formation time discussion we had earlier. The non-vanishing $v_{2}$ at high $p_{t}$ is mainly due to fluid-jet interactions, so the values should be related to the estimated probability $P_{\text {inside }}$ to form the jet hadron inside the fluid, which is equivalent to the fluid-jet interaction probability. In Fig. 33, we show $P_{\text {inside }}$ (multiplied by an arbitrary factor), together with the calculated and experimental $v_{2}$ already show in Fig. 32. which shows that $v_{2}$ is indeed proportional to the fluid-jet interaction probability. To compute $P_{\text {inside }}$ according to eq. (5), we use $c \tau_{\text {form }}=1 \mathrm{fm}, m c^{2}=1 \mathrm{GeV}$, $r_{\mathrm{Pb}}=6.5 \mathrm{fm}$, and $b=11.5 \mathrm{fm}(50-60 \%)$. 


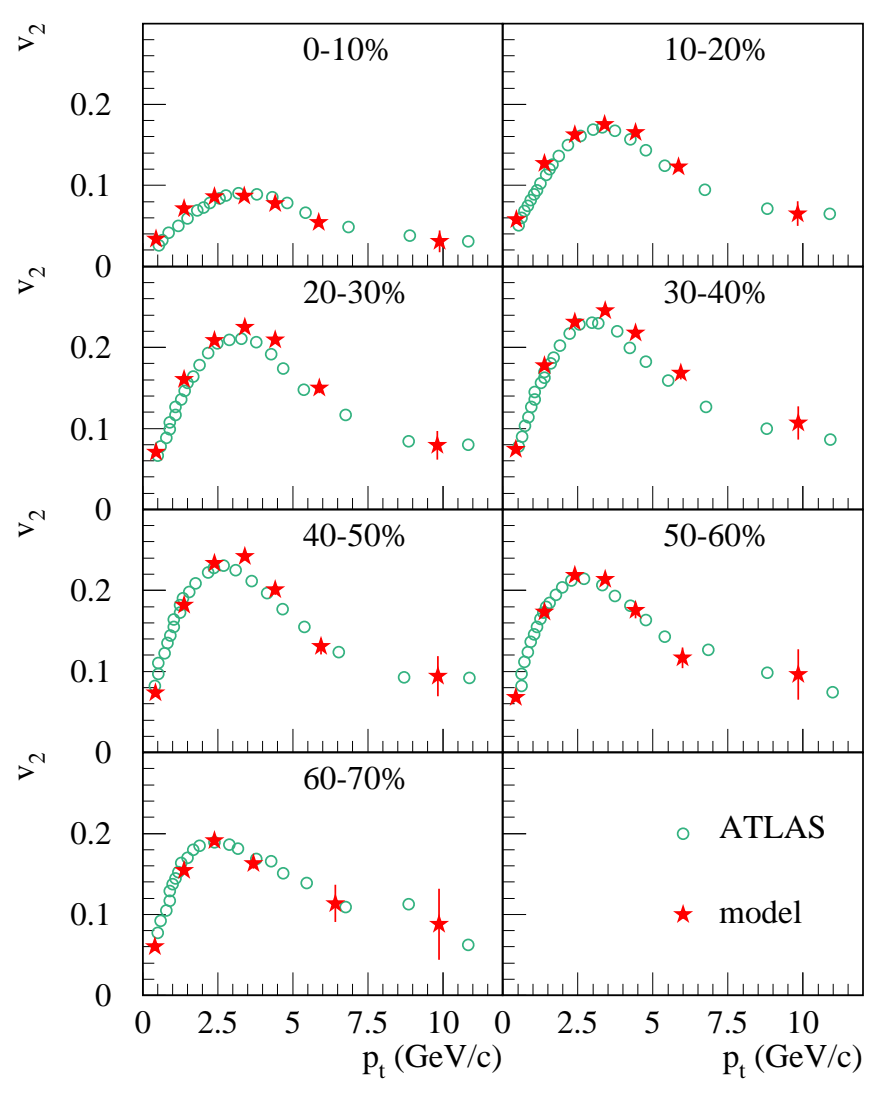

Figure 32: (Color online) $p_{t}$ dependence of elliptical flow (defined with respect to the opposite hemisphere sub-event plane) for different centralities in $\mathrm{Pb}-\mathrm{Pb}$ collisions at $2.76 \mathrm{TeV}$. We compare the ATLAS data [6] (circles) with calculations (red lines).

Having a finite $P_{\text {inside }}$ is a necessary condition to get a substantial $v_{2}$ at some large value of $p_{t}$, but not a sufficient one. Let us therefore estimate the effect of the fluidjet interaction on $v_{2}$. One may consider a toy model, with soft particle emission due to flow into some preferred azimuthal direction, say $\phi_{\text {flow }}$. Let us assume a jet hadron getting pushed by the fluid in the direction of $\phi_{\text {flow }}$, which corresponds to adding some $\vec{p}_{t}^{\text {soft }}$ to the "hard" transverse momentum $\vec{p}_{t}^{\text {hard }}$ of the flux-tube segment. Without loss of generality, we may set $\phi_{\text {flow }}=0$. The total transverse momentum of the jet hadron is

$\vec{p}_{t}^{\text {soft }}+\vec{p}_{t}^{\text {hard }}=\left(\begin{array}{c}p_{t}^{\text {soft }}+p_{t}^{\text {hard }} \cos (\phi) \\ p_{t}^{\text {hard }} \sin (\phi)\end{array}\right)=p_{t}^{\text {jet }}\left(\begin{array}{c}\cos \psi \\ \sin \psi\end{array}\right)$,

where $\psi$ is the jet hadron direction with respect to the flow direction $\phi_{\text {flow }}=0$. We have

$$
\tan \psi=\frac{p_{t}^{\text {hard }} \sin (\phi)}{p_{t}^{\text {soft }}+p_{t}^{\text {hard }} \cos (\phi)} .
$$

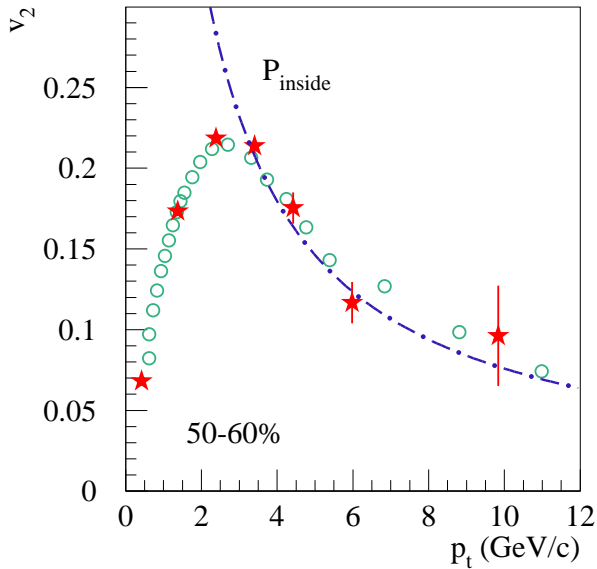

Figure 33: (Color online) Same data and model calculation as shown in Fig. 32 for the case of 50-60\% most central collisions, together with $P_{\text {inside. }}$.

Assuming a flat $\phi$ distribution, the probability distribution for $\psi$ is $(2 \pi)^{-1} d \phi / d \psi$, which is in the case of $p_{t}^{\text {soft }} \ll p_{t}^{\text {hard }}$ given as

$$
1+\frac{p_{t}^{\text {soft }}}{p_{t}^{\text {hard }}} \cos (\psi)
$$

which should only be considered for $-\pi / 2<\psi<\pi / 2$, since $\psi$ and $\phi_{\text {flow }}$ have to correspond to the same hemisphere. We get anisotropies of the order of $p_{t}^{\text {soft }} / p_{t}^{\text {hard }}$, which means at transverse momenta around $10 \mathrm{GeV} / \mathrm{c}$, a soft push as little as $1 \mathrm{GeV} / \mathrm{c}$ can produce anisotropies of the order of $10 \%$.

\section{COALESCENCE}

For many years, different models have been employed to treat particle production at different transverse momentum scales. The so-called intermediate range from 2 to $6 \mathrm{GeV} / \mathrm{c}$ has been the domain of coalescence models [29 33], where hadrons are produced by recombining quarks from the plasma, to be distinguished from "fragmentation" of partons.

In our picture, there are certain aspects which give similar results as coalescence, but it is not a coalescence approach. Already the notion "intermediate $p_{t}$ " extends to say $20 \mathrm{GeV} / \mathrm{c}$ and not 6 . The corresponding transverse momentum of hadrons does not originate from plasma quarks and antiquarks - the main part is coming from the original flux tube. Whereas usual flux tube breaking in vacuum creates quark-antiquark pairs via a tunneling process, the fluid-jet interaction amounts to replacing these quark-antiquark pairs by partons from the plasma. 
So our jet hadrons finally carry "some" transverse momentum from fluid partons, but only a small fraction. But this is enough to create for example anisotropies in dihadron correlations. It will also affect strongly baryon to meson rations, as we are going to discuss in a separate publication.

\section{SUMMARY}

We presented a theoretical scheme which accounts for bulk matter, jets, and the interaction between the two. The criterion for bulk-jet separation is based on parton energy loss. But in addition to the latter mechanism, there are very important new phenomena which have not been discussed so far: The interaction between jet hadrons and soft ones (from fluid freeze-out), and the interaction between the fluid and jet hadrons at the moment of the creation of the latter ones. Particle production between zero and (at least) $20 \mathrm{GeV} / \mathrm{c}$ is affected. We understand quantitatively azimuthal anisotropies in single particle production and dihadron (long range) correlations at large values of $p_{t}$.

\section{Acknowledgments}

This research has been carried out within the scope of the ERG (GDRE) "Heavy ions at ultra-relativistic energies", a European Research Group comprising IN2P3/CNRS, Ecole des Mines de Nantes, Universite de Nantes, Warsaw University of Technology, JINR Dubna, ITEP Moscow, and Bogolyubov Institute for Theoretical Physics NAS of Ukraine. Iu.K. acknowledges partial support by the State Fund for Fundamental Researches of Ukraine (Agreement of 2011) and National Academy of Sciences of Ukraine (Agreement of 2011). K.W. would like to thank Ludmila Malinina and Konstantin Mikhailov for useful discussions. M.B. thanks the Hessian LOEWE initiative for financial support.
[1] STAR Collaboration, B. Abelev et al., Phys. Rev. C 80 (2009) 64192S

[2] PHENIX collaboration, A.Adare et al., Phys.Rev.C78:014901,2008

[3] PHOBOS collaboration, B. Alver et al., Phys. Rev. Lett. 104, 062301 (2010)

[4] CMS Collaboration, S. Chatrchyan et al., JHEP 1107 (2011) 076, arXiv:1105.2438; arXiv:1201.3158

[5] ALICE collaboration, K. Aamod et al., Phys.Lett. B708 (2012) 249-264, arXiv:1109.2501

[6] ATLAS collaboration, G. Aad et al., Phys.Lett. B707 (2012) 330-348, arXiv:1108.6018

[7] HS06 is the abbreviation for HEP-SPEC-2006, which is a benchmark for measuring CPU performances. The machines we use have an average power of 8.7 HS06 units, this number being referred to as "scaling factor".

[8] K. Werner, Iu. Karpenko, T. Pierog, M. Bleicher, K. Mikhailov, Phys. Rev. C 82, 044904 (2010)

[9] H. J. Drescher, M. Hladik, S. Ostapchenko, T. Pierog and K. Werner, Phys. Rept. 350, 93, 2001

[10] Y. Nambu, Proc. Intl. Conf. on Symmetries and Quark Models, Wayne State Univ., 1969

[11] J. Scherk, Rev. Mod. Phys. 47, 123 (1975)

[12] C. Rebbi, Phys. Rep. 12 (1974) 1

[13] X. Artru, Phys. Rep. 97, 147 (1983)

[14] D. A. Morris, Nucl. Phys. B288, 717, 1987

[15] K. Werner, Iu. Karpenko, T. Pierog, M. Bleicher, K. Mikhailov, arXiv:1010.0400, Phys. Rev. C 83, 044915 (2011)

[16] K. Werner, Iu. Karpenko, T. Pierog, arXiv:1011.0375, Phys. Rev. Lett. 106, 122004,2011

[17] M. Cacciari, G. Salam, and G. Soyez, JHEP 0804 (2008) 063
[18] ATLAS collaboration, Aad et al., Eur. Phys. J. C71 (2011) 1512

[19] S. Kretzer, H.L. Lai, F. Olness, W.K. Tung, Phys. Rev. D69:114005(2004)

[20] R. Baier et al., Nucl.Phys.B483:291-320,1997; S. Peigné, AIP Conf.Proc.1038:139-148,2008

[21] M. Bleicher et al., J. Phys. G25 (1999) 1859; H. Petersen, J. Steinheimer, G. Burau, M. Bleicher and H. Stocker, Phys. Rev. C78 (2008) 044901

[22] R. Bellwied \& C. Markert, PLB 691 (2010) 208 and arXiv:1005.5416

[23] S. Borsanyi et al., JHEP 1011 (2010) 077, arXiv: 1007.2580

[24] CMS collaboration, S. Chatrchyan et al., JHEP 1108,141, arXiv: 1107.4800

[25] ATLAS collaboration, G. Aad et al., Phys.Lett. B710 (2012) 363-382, arXiv:1108.6027

[26] R. Preghenella, ALICE collaboration, Acta Phys.Polon. B43 (2012) 555, arXiv:1111.7080

[27] K. Gallmeister, C. Greiner, Z. Xu, Phys.Rev.C67, 044905, 2003; W. Cassing, K. Gallmeister, C. Greiner, Nucl.Phys.A735:277-299,2004.

[28] ALICE collaboration, K. Aamod et al., Phys.Lett. B696 (2011) 30-39, arXiv:1012.1004

[29] D. Molnar and S. A. Voloshin, Phys. Rev. Lett. 91, 092301 (2003).

[30] V. Greco, C. M. Ko, and P. Levai, Phys. Rev. Lett. 90, 202302 (2003); Phys. Rev. C 68, 034904 (2003).

[31] R. J. Fries, B. Mueller, C. Nonaka, and S. A. Bass, Phys. Rev. Lett. 90, 202303 (2003); Phys. Rev. C 68, 044902 (2003).

[32] R. C. Hwa and C. B. Yang, Phys. Rev. C 67, 034902 (2003); Phys. Rev. C 70, 024905 (2004). 
[33] S. Pratt, S. Pal, Phys.Rev. C71 (2005) 014905. 